\title{
The selfish brain
}

\author{
O cérebro egoísta
}

Roger S. McIntyre*

Drs. Mansur and Brietzke proffer a conceptual framework that attempts to reconcile disparate physiological processes as part of a broader aim to inform pathoetiological models in bipolar disorder (BD) and schizophrenia (SCZ). Tacit to their proposal is that the existing models of disease pathoetiology (e.g., the monoamine hypothesis) are insufficient at explaining the melange of phenomenology encountered in these severe lifelong illnesses. Moreover, existing models fail to provide a compelling explanation for observations of "progression" in mental illness. A central motif of their framework is that alterations in bioenergetics are causative (and possibly consequential) of these highly familial and heritable conditions.

Their proposal is propitious in its timing, as a fundamental reconceptualization of mental disorders is occurring. More specifically, it is proposed that mental disorders can be conceptualized as a developmental process gone awry. That is, normal organism development goes "off trajectory" as a result of a concatenation of vulnerability, environmentally pathogenic, and resiliency factors. Seventy-five per cent of all mental disorders begin before the age of 25 . A highly replicated observation in clinical psychiatry is a more favourable course and outcome with earlier detection, diagnosis, treatment, and management. A derivative of this observation is that treatment response to either pharmacotherapy and/or psychosocial interventions diminishes as a function of episode frequency. Taken together, these observations implicate a psychobiological progression of illness with preliminary evidence of "staging." 1

The pathoetiology of BD and SCZ, like that of other chronic medical disorders (e.g., type II diabetes mellitus, cardiovascular disease), is multifactorial. These disorders do not exhibit Mendelian inheritance patterns; instead, evidence indicates that vulnerability to $B D$ and SCZ is a consequence of numerous (dozens, if not hundreds to thousands of) vulnerability genes, each with a relatively low effect size and lacking specificity for any mental (or medical) disorder. Epigenetic modifications as a result of exposure to environmental pathogens during critical periods of development influence the transcription and conformational phenotypes of vulnerability and resiliency genes. A well known strategic approach that is currently embraced in many chronic diseases is the pursuit of "omics," with efforts underway to characterize genomic, metabolomic, proteomic, transcriptomic profiles, as well as connectomics, relevant to the disease process. Recent evidence identifying a relationship between common cold infection and the onset of type II diabetes mellitus with dynamic effects on omic profiles using an integrated personal omics profiling (iPOP) approach serves as a useful lesson and exemplar for psychiatry. ${ }^{2}$

Reductionistic models implicating single determinants of disease causation have strength in their simplicity, but are highly limited in their explanatory power. One could in fact be somewhat surprised at how enduring and fruitful the monoamine hypothesis has been in neuropsychiatric disorders. Psychiatry has clearly been suffering from paradigm strain, as evidenced by the relative absence of any genuinely novel, innovative pharmacological approaches for the treatment of mood and psychotic disorders in over 50 years. The drug discovery process is stale dated and has been unable to satisfy the unmet needs in the clinical ecosystem at best, and at worst has served as convenient fodder for anti-psychiatry

\footnotetext{
* MD, FRCPC. Professor of Psychiatry and Pharmacology, University of Toronto, Toronto, ON, Canada. Head, Mood Disorders Psychopharmacology Unit, University Health Network, Toronto, ON, Canada. E-mail: roger.mcintyre@uhn.ca No conflicts of interest declared concerning the publication of this editorial. Suggested citation: McIntyre RS. The selfish brain. Trends Psychiatry Psychother. 2012;34(3):119-20.
} 
organizations (as well as other sceptics) of the scientific edifice of psychiatry.

The notion that bioenergetics is central to disease pathoetiology in neuropsychiatric disorders discussed by Drs. Mansur and Brietzke is highly supported by preclinical, post-mortem, cellular, molecular, pharmacological, as well as clinical data. The evolutionary advantage of the "selfish brain" has a face validity that is undeniable with examples of "selfish brains" in other clinical scenarios (e.g., asymmetrical intrauterine growth restriction).

There is much work to be done to reify or refute the "selfish brain" concept. As a starting point and cliché, more descriptive work is needed, however with an encouragement to take an iPOP approach. I suspect Drs. Mansur and Brietzke would surmise that metabolomics would be a critical part of any iPOP (I would agree!). There is an urgent need to identify molecular targets for interventional research to assist the field with the necessary empiricism and, consequently, genuinely novel and viable treatments based on disease pathoetiology. The phlegmatic pace of drug discovery in psychiatry has indeed its beneficiaries. Optimistically, novel approaches will also have their beneficiaries. It is exciting to consider that tectonic plate shifts will occur in how the field conceptualizes disease pathoetiology, prevention, and treatment.

We are continuously reminded that the syndromes encountered in psychiatry represent a final common pathway of abnormalities in cells, synapses, and circuits. It is no longer plausible that a single pharmaceutical approach will ameliorate all dimensions of psychopathology. I would put forth a modified hypothesis that perhaps disturbances in bioenergetics, as exemplified by the "selfish brain," may in fact provide greater insight into the determinants of domains of psychopathology (e.g., cognitive function) rather than the full syndrome.

I applaud Drs. Mansur and Brietzke for shifting us from the pre-contemplative to the contemplative and for inspiring us toward action. The pathoetiological nexus that they implicate could possibly extend our understanding as to why individuals with mental illness are differentially affected by metabolic-related medical comorbidity. For me, an important unanswered question is the direction of causality (e.g., are bioenergetic disturbances primary or epiphenomenon?). Reconciling my own clinical observations of high rates of medical disorders in the persistently mentally ill along with extant evidence leads me to conclude that in some cases mental disorders begin in the brain and metastasize to the body, while perhaps in other cases it may begin in the body and metastasize to the brain.

\section{References}

1. Kapczinski F, Vieta E, Andreazza AC, Frey BN, Gomes FA, Tramontina J, et al. Allostatic load in bipolar disorder: implications for pathophysiology and treatment. Neurosci Biobehav Rev. 2008;32:675-92.

2. Chen R, Mias GI, Li-Pook-Than J, Jiang L, Lam HY, Chen R, et al. Personal omics profiling reveals dynamic molecular and medical phenotypes. Cell. 2012;148:1293-307. 